\title{
Aislamiento e Identificación de Bacterias y Levaduras Resistentes a Petróleo
}

\author{
Ismael Acosta-Rodríguez ${ }^{(1)}$, María G. Moctezuma-Zárate ${ }^{(1)}$, Juana Tovar-Oviedo ${ }^{(2)}$ y \\ Juan F. Cárdenas-González ${ }^{(1)}$ \\ Universidad Autónoma de San Luis Potosí, Facultad de Ciencias Químicas, Centro de \\ Investigación y de Estudios de Posgrado, (1) Lab. de Micología Experimental; (2) Lab. de \\ Microbiología, Av. Dr. Manuel Nava No. 6, Zona Universitaria, 78320 San Luis Potosí, S.L.P.- \\ México. (e-mail: iacosta@uaslp.mx)
}

Recibido Feb. 20, 2011; Aceptado Abr. 26, 2011; Versión Final recibida May. 24, 2011

\section{Resumen}

Se aislaron diferentes microorganismos de varios ríos de la Huasteca Potosina en México, los cuales crecen en presencia de petróleo como única fuente de carbono. Se determinó la actividad de alcohol oxidasa por un método colorimétrico. La bacteria más frecuentemente encontrada fue Pseudomonas aeruginosa (50\%) y se encontró solamente una levadura, Candida albicans $(6.25 \%)$. Además, presentan buena actividad de alcohol oxidasa en la fracción citosólica con diferentes sustratos. Se concluye que estos microorganismos pueden ser utilizados para la eliminación y degradación de petróleo en sitios contaminados.

Palabras clave: microorganismos, bacterias, contaminación por petróleo, Candida albicans, alcohol oxidasa

\section{Isolation and Identification of Petroleum Resistant Bacteria and Yeast}

\begin{abstract}
Different microorganisms were isolated from various rivers of the Huasteca Potosina, in Mexico that grow in the presence of petroleum as the sole carbon source. The activity of alcohol oxidase was determined by a colorimetric method. The most common bacteria present in the samples were Pseudomonas aeruginosa (50\%) and only one type of yeast was found, Candida albicans (6.25\%). Furthermore, they show good activity of alcohol oxidase in the cytosolic fraction with different substrates. It was concluded that this microorganisms could be used for decontamination of aquatic habitats polluted with petroleum.
\end{abstract}

Keywords: microorganisms, bacteria, petroleum pollution, Candida albicans, alcohol oxydase 


\section{INTRODUCCIÓN}

Los derrames de petróleo son una importante fuente de contaminación del suelo y agua, ya que el uso, más el transporte transfronterizo tanto de petróleo crudo como de sus derivados, derrames de contenedores, rupturas en tuberías subterráneas y diferentes procesos industriales, hace que los derrames de hidrocarburos sean cada vez más frecuentes, lo que provoca riesgos asociados a la salud humana por la inhalación de vapores y la ingestión de aquellos hidrocarburos que están disueltos en el agua y el contacto dérmico, que se da principalmente en actividades recreativas, pues algunos de sus componentes son considerados carcinogénicos y teratogénicos (Chen y Liao, 2006). También origina que se desarrolle tolerancia a la presencia de este compuesto, induciendo la selectividad y la disminución de la diversidad microbiana en los diferentes nichos ecológicos contaminados. Los microorganismos tolerantes a petróleo, desarrollan y utilizan diferentes respuestas especializadas (enzimáticas y fisiológicas) para crecer en presencia de este contaminante (Atlas et al., 1991). Estas condiciones propician las variaciones poblacionales de los microorganismos autóctonos, y de manera natural realizan la degradación química del petróleo presente en aguas y suelos.

El petróleo crudo contiene cientos de compuestos individuales, pero presenta cuatro formas estructurales en función de la solubilidad en solventes orgánicos: compuestos saturados (alcanos y cicloparafinas), aromáticos (mono, di y polinúcleo aromáticos), resinas (agregados con una gran cantidad de estructuras como: piridinas, quinolinas, carbazoles, tiofenos, sulfóxidos y aminas) y asfaltenos (agregados de poliaromáticos, ácidos nafténicos, fenoles, ácidos grasos y metaloporfirinas) (Leahy y Colwell, 1990).

Se ha demostrado que el crecimiento de los microorganismos requiere de fuentes de carbono derivadas de los hidrocarburos del petróleo. Los compuestos saturados y los aromáticos con uno a cinco anillos bencénicos son utilizados como fuentes energéticas; sin embargo, los aromáticos con más de cinco anillos, resinas y asfaltenos son difíciles de degradar por su recalcitrancia (Sugiera et al., 1997). La selección de microorganismos a través de pruebas sucesivas de crecimiento poblacional en cultivos puros ricos en petróleo, es una estrategia eficiente para evaluar la adaptación y sobrevivencia de cepas tolerantes a altas concentraciones de petróleo. Los resultados de las pruebas en laboratorio confirman la selección de las cepas más tolerantes y adaptadas. El éxito en las siguientes etapas, tanto en invernadero como en suelos y aguas contaminadas, depende de la calidad de la selección y de las condiciones ambientales (Rivera Cruz et al., 2002). Es muy importante la evaluación sucesiva de los microorganismos que utilizan hidrocarburos derivados del petróleo como fuente de energía, para demostrar la eficiencia de las tecnologías de biorremediación en suelos y aguas expuestas a concentraciones tóxicas de petróleo (Martín Moreno et al., 2004).

Recientemente, se ha estudiado el aislamiento de microorganismos tolerantes y su capacidad de degradación, a partir de sitios contaminados con el mismo, como la bacterias Rhodococcus aetherivorans y E. wratislaviensis (Auffret et al., 2009), Streptomyces spp (Saadoun et. al.,) y Pseudomonas aeruginosa sp (Tang et al., 2007; Emtiazi et al., 2005), las levaduras Saccharomyces cerevisiae y Candida albicans (llori et. al., 2008), y los hongos filamentosos Penicillium sp y Aspergillus sp (Rivera-Cruz et al., 2002) Trichoderma asperellum (Husaini et al., 2008). Por lo anterior el objetivo de este trabajo fue el aislamiento e identificación de bacterias tolerantes a petróleo crudo a partir de algunos ríos de la Huasteca Potosina.

\section{METODOLOGÍA}

\section{Muestras de agua}

Se tomaron en recipientes de plástico previamente lavados con ácido sulfúrico al $10 \%$ y esterilizados por calor húmedo, muestras de agua $(500 \mathrm{~mL})$, de algunos ríos de la Huasteca Potosina: Coy, Amajac, Tamazunchale, Valles, Tamuín, Bancote, La galera y Santa Rosa, durante el periodo julio-septiembre de 2010. Se guardaron en hielera, y se trasladaron al laboratorio y se conservaron en refrigeración hasta su uso. 


\section{Aislamiento e identificación de las cepas bacterianas}

El aislamiento se realizó inoculando $1.0 \mathrm{~mL}$ de las muestras de agua en cajas de Petri conteniendo medio mínimo de Lee (Lee et al., 1975), sin glucosa y adicionadas de $1.0 \mathrm{~mL}$ de petróleo como fuente de carbono, incubando a $28^{\circ} \mathrm{C}$ durante 5 días. Las colonias obtenidas se purificaron por resiembras sucesivas en el mismo medio de cultivo, y para su posterior identificación, se sembraron por duplicado en los siguientes medios selectivos: Agar hierro de Kligler, SIM (Sulfhídrico-Indol-Movilidad) y OF (oxidación-fermentación) para Pseudomonas, así como Agar Biggy y la prueba de tubo germinal para levaduras (López Martínez et al., 2004). También se realizó toda la batería de pruebas bioquímicas para la identificación de Enterobacterias (API 20 E) (Koneman, et al., 2002).

\section{Estudios de Resistencia a Petróleo}

La resistencia se analizó inoculando $1 \times 10^{6}$ bacterias y/o levaduras $/ \mathrm{mL}$ en matraces Erlenmeyer de $250 \mathrm{~mL}$ conteniendo $100 \mathrm{ml}$ de medio mínimo de Lee, conteniendo $1.0 \mathrm{~mL}$ de petróleo como fuente de carbono, incubando a $28^{\circ} \mathrm{C}$ a $100 \mathrm{rpm}$ durante 3 días para las bacterias y 7 días para las levaduras. Después, se cosechó el sobrenadante en un tubo graduado, previamente pesado y se centrífugo a $3000 \mathrm{rpm} / 10 \mathrm{~min}$, desechando el sobrenadante. El paquete celular se seco a $80^{\circ} \mathrm{C}$, durante $4 \mathrm{~h}$, y se peso el tubo, determinando por diferencia el peso seco de la muestra, comparando el crecimiento con un control crecido en las mismas condiciones sin la adición de petróleo crudo. Todos los experimentos se realizaron mínimo 3 veces por duplicado.

\section{Obtención del extracto libre de células}

Se inocularon $1 \times 10^{6}$ bacterias y/o levaduras $/ \mathrm{mL}$ en matraces Erlenmeyer de $250 \mathrm{~mL}$ conteniendo $100 \mathrm{ml}$ de medio mínimo de Lee, con y sin $1.0 \mathrm{~mL}$ de petróleo crudo, incubando a $28^{\circ} \mathrm{C}$ a $100 \mathrm{rpm}$ durante 3 días para las bacterias y 7 días para las levaduras, obteniendo el paquete celular de cada cultivo filtrando en papel Whatman No.1 y lavando por centrifugación (2 $000 \mathrm{rpm}$ ) las células con agua destilada estéril fría (un promedio 3 veces o las necesarias hasta que el líquido sobrenadante sea claro). Posteriormente, el paquete celular se resuspendió en $1.0 \mathrm{~mL}$ de agua destilada estéril fría, y se añaden $4.0 \mathrm{~mL}$ de solución amortiguadora de rompimiento (Tris- $\mathrm{HCl} 50$ mM. pH 8.5; PMSF $1 \mathrm{mM}$, disuelto en dimetilsulfóxido), realizando el rompimiento de la masa celular mediante Omni-mixer y Potter, manteniendo el homogenado en hielo para mantener la temperatura lo más baja posible. Después, el paquete celular se centrifugó a 3000 rpm, durante 15 min para remover las paredes celulares y las células no rotas. El sobrenadante (extracto crudo) se centrifugó a $25000 \mathrm{rpm}$ por $45 \mathrm{~min}$, descartando la fracción mixta de membranas (FMM) y al sobrenadante se le determinó la actividad de alcohol oxidasa.

\section{Determinación de la actividad de alcohol oxidasa por un método colorimétrico}

Se cuantifican los micromoles de peróxido de hidrógeno formados por minuto por miligramo de proteína (actividad específica), debido a la oxidación del alcohol catalizado por la enzima alcohol oxidasa. La actividad total se define como el producto de la actividad específica por la proteína total (Jannsen et al., 1975).

\section{Reactivos}

${ }^{*}$ Regulador de fosfato de potasio $0.2 \mathrm{M} \mathrm{pH} 7.5$

${ }^{*}$ Dihidrocloruro de O-dianisidina al 1\% disuelto en $\mathrm{HCl} 25 \mathrm{mM}$. SIGMA CHEMICAL CO.

${ }^{*} \mathrm{H}_{2} \mathrm{O}_{2} 0.3 \%$

${ }^{*}$ Reactivo A: $1.2 \mathrm{~mL}$ de regulador de fosfato de potasio $0.2 \mathrm{M} \mathrm{pH} 7.5$, agregar $10 \mu \mathrm{L}$ de $\mathrm{O}$ dianisidina al $1 \%$.

Sustratos (concentración final):

*Metanol absoluto $127 \mathrm{mM}$.

*Etanol absoluto $127 \mathrm{mM}$

*Petróleo crudo $0.015 \mathrm{p} / \mathrm{v}$ 


\section{Mezcla de reacción.}

$780 \mu \mathrm{L}$ de reactivo $\mathrm{A}$

$5 \mu \mathrm{L}$ peroxidasa $0.01 \%$ (concentración final).

$150 \mu \mathrm{L}$ de regulador de fosfato de potasio $0.2 \mathrm{M}$

$15 \mu \mathrm{L}$ de sustrato

$50 \mu \mathrm{L}$ de extracto enzimático.

La reacción se inicia por la adición del sustrato, y se incuba a $28^{\circ} \mathrm{C}$ durante $30 \mathrm{~min}$. El desarrollo de color (formación de $\mathrm{H}_{2} \mathrm{O}_{2}$ ) se determinó por la lectura de la absorbancia a $460 \mathrm{~nm}$. Se realizó una curva patrón con solución de peróxido de hidrogeno a diferentes concentraciones.

\section{Determinación de Proteína}

Se empleó el método descrito por Lowry et al., (1951), usando como patrón albúmina de suero bovino (ASB).

\section{RESULTADOS Y DISCUSIÓN}

A partir de las diferentes muestras de río analizadas, se aislaron e identificaron 15 colonias de bacterias y una levadura capaces de crecer en presencia de petróleo (Tabla No. 1), siendo la más frecuente Pseudomonas aeruginosa (50.0\%), seguida de Escherichia coli (31.25\%) y Enterobacter aerogenes, Proteus mirabilis y Candida albicans (6.25\%) (Figura No.1), lo que indica que los microorganismos aislados desarrollaron la resistencia y tal vez el mecanismo de degradación del petróleo en un medio ambiente contaminado con el mismo, lo cual coincide con una gran variedad de estudios, pues a partir de diferentes fuentes, se han aislado diferentes microorganismos con la capacidad de resistencia y degradación del petróleo (Auffret et al., 2009; Saadoun et. al., 2008; Tang et al., 2007; Emtiazi et al., 2005; llori et. al., 2008; Rivera-Cruz et al., 2002; Husaini et al., 2008).

Tabla 1: Colonias de bacterias identificadas en las muestras analizadas. * Levadura

\begin{tabular}{|l|l|l|}
\hline Fuente (Río) & Colonia identificada & Total \\
\hline Amajac-1 & Pseudomonas aeruginosa & 1 \\
\hline Amajac-2 & $\begin{array}{l}\text { Pseudomonas aeruginosa } \\
\text { Enterobacter aerogenes }\end{array}$ & 2 \\
\hline Bancote-1 & $\begin{array}{l}\text { Candida albicans * } \\
\text { Escherichia coli } \\
\text { Proteus mirabilis }\end{array}$ & 3 \\
\hline Bancote-2 & Escherichia coli & 1 \\
\hline Valles, Planta tratadora-1 & Pseudomonas aeruginosa & 1 \\
\hline Valles, Planta tratadora-2 & Pseudomonas aeruginosa & 1 \\
\hline Valles, Calera-2 & Pseudomonas aeruginosa & 1 \\
\hline Coy, Planta tratadora & Pseudomonas aeruginosa & 1 \\
\hline Amajac/Moctezuma-1 & Pseudomonas aeruginosa Escherichia coli & 2 \\
\hline Amajac/Moctezuma-2 & Escherichia coli & 1 \\
\hline Santa Rosa-1 & Escherichia coli & 1 \\
\hline Santa Rosa-2 & Pseudomonas aeruginosa & 1 \\
\hline
\end{tabular}




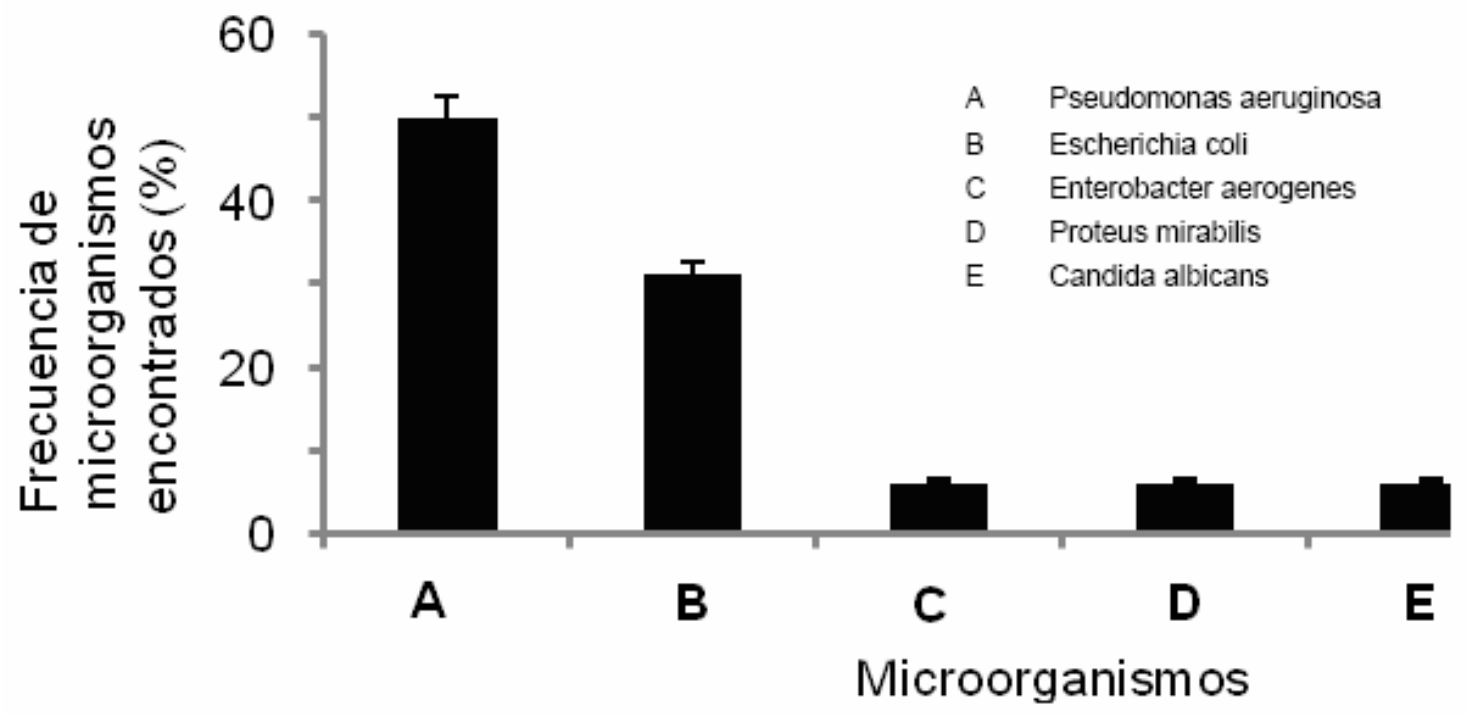

Fig. 1: Frecuencia de microorganismos encontrados

Posteriormente, se incubaron en presencia de $1.0 \mathrm{~mL}$ de petróleo crudo las ocho cepas aisladas de $P$. aeruginosa (3 días) y $C$. albicans (7 días), y se les determinó el crecimiento por peso seco, encontrando que todas las bacterias crecen mejor en presencia del hidrocarburo, presentando mayor y menor crecimiento la $P$. aeruginosa aislada del Río Coy, y la del Río Valles (Calera) con un crecimiento de 4.5 (139 $\mathrm{mg}$ de peso seco) y 1.0 veces (34 $\mathrm{mg}$ de peso seco) respectivamente, (Figura No. 2), mientras que la levadura también crece mejor cuando se adicionan al medio de cultivo diferentes concentraciones de petróleo crudo $(200-1000 \mu \mathrm{L})$, con un rango de crecimiento promedio de 1.3 veces y entre 39 y $42.5 \mathrm{mg}$ de peso seco, con respecto al control (Figura No. 3). Se ha encontrado que el $96 \%$ de bacterias aisladas de medios líquidos (lagos, ríos, y lagunas) presentan capacidad de crecen y emulsificar hidrocarburos derivados del petróleo (Leahy y Colwell, 1990), y los resultados obtenidos en este trabajo, demuestran que todas las colonias de $P$. aeruginosa y de la levadura $C$. albicans obtenidas, crecen eficientemente en el medio líquido adicionado con $1.0 \mathrm{~mL}$ de petróleo crudo, además de emulsificar el medio de cultivo. Estos resultados son similares a los obtenidos por Rosenberb et al., (1992) con cepas Gram negativas puras, Auffret et al., (2009) con las bacterias R. aetherivorans y E. wratislaviensis, Tang et al., (2007); Emtiazi et al., (2005); Mittal y Singh (2009) con P. aeruginosa y sp. y con llori et. al., (2008) para la levadura $C$. albicans. La sobrevivencia de las bacterias y la levadura en estas condiciones, sugiere que podrían tener la capacidad de utilizar hidrocarburos alifáticos y aromáticos como fuentes de carbono y/o donadores de electrones (Martín Moreno et al., 2004; Argumedo-De Lira et al., 2009).

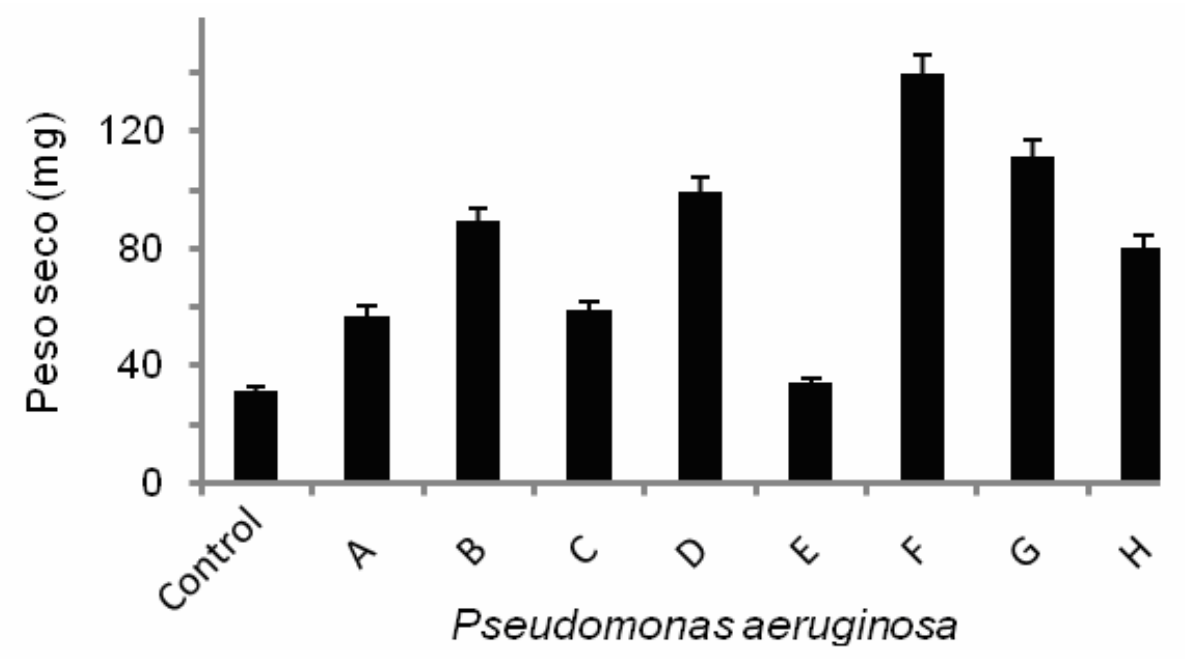

Fig. 2: Crecimiento en peso seco de Pseudomonas aeuroginosa en el medio de cultivo adicionado de $1 \mathrm{~mL}$ de petróleo crudo. $28^{\circ} \mathrm{C}, 100 \mathrm{rpm}$. 


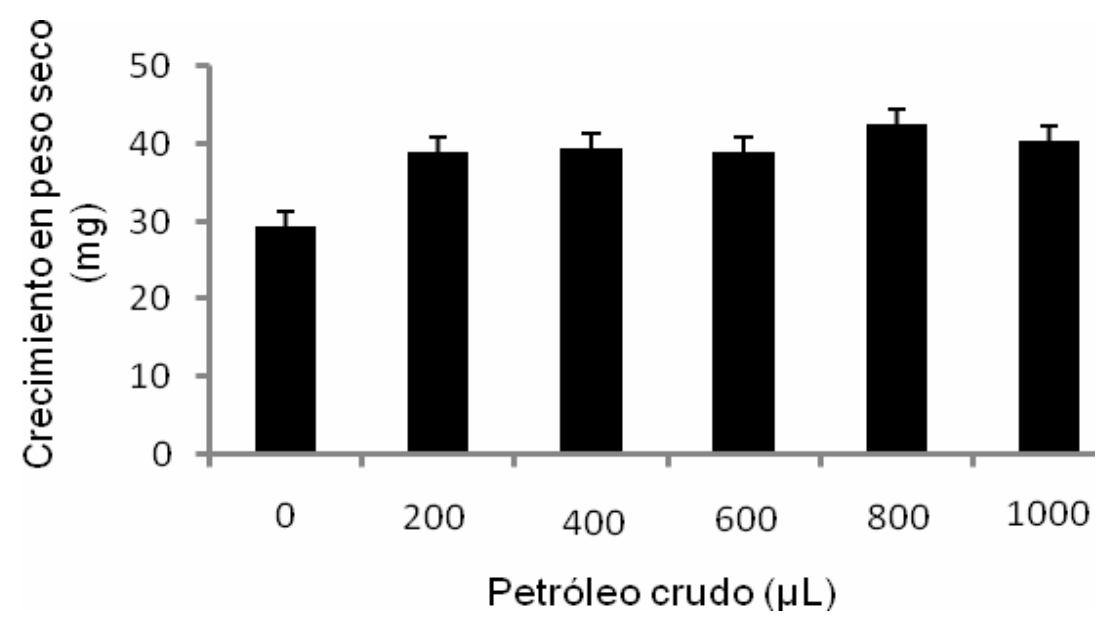

Fig. 3: Crecimiento en peso seco de Candida albicans en el medio de cultivo adicionado de diferentes concentraciones de petróleo crudo. $28^{\circ} \mathrm{C}, 100 \mathrm{rpm}$.

También, se analizó la actividad de alcohol oxidasa en las diferentes fracciones subcelulares (extracto crudo, FMM y sobrenadante de $25000 \mathrm{rpm}$ ) utilizando como sustrato petróleo crudo, metanol y etanol, de la cepa de $P$. aeruginosa aislada del Río Coy (qué fue la que mostró mayor crecimiento) y de $C$. albicans (única levadura aislada), crecidas en presencia y ausencia de petróleo crudo (ver Metodología). La actividad enzimática con los 3 sustratos utilizados, se detectó principalmente en la fracción citosólica, y poca en la FMM (datos no mostrados). La Tabla 2 muestra los niveles de actividad específica de ambas cepas utilizadas, siendo mayor cuando se crecen en presencia de petróleo y metanol como sustrato (345 para $P$. aeruginosa y de 279 para C. albicans), aunque con petróleo crudo la levadura muestra muy buena actividad enzimática (175.8). Los resultados encontrados en este trabajo son similares a los reportados por AlvaradoCaudillo et al., (2002) para el hongo YR-1 aislado de suelos contaminados con petróleo, aunque ellos utilizan 11 sustratos diferentes, siendo el metanol el principal inductor enzimático, y con los de Durón-Castellanos et al., (2005) para la alcohol deshidrogenasa dependiente de NAD+ con metanol, etanol y hexadecanol como sustratos y son diferentes a lo reportado por Silva-Jiménez et al., (2009) para una alcohol oxidasa grasa de Mucor circinelloides YR-1 con metanol, decanol y hexadecanol como sustratos.

Tabla 2: Actividad de alcohol oxidasa en Pseudomonas aeruginosa y Candida albicans crecidas con y sin petróleo crudo.

\begin{tabular}{|l|c|c|c|}
\hline Sustrato & Petróleo $(\mathrm{mL})$ & $\begin{array}{c}\text { Pseudomonas aeruginosa } \\
\text { Actividad especifica } \\
\left(\mu \mathrm{H} \mathrm{H}_{2} \mathrm{O}_{2} / \mathrm{min} / \mathrm{mg} \text { proteina }\right)\end{array}$ & $\begin{array}{c}\text { Candida albicans } \\
\text { Actividad especifica } \\
\left(\mu \mathrm{g} \mathrm{H}_{2} \mathrm{O}_{2} / \mathrm{min} / \mathrm{mg} \text { proteina }\right)\end{array}$ \\
\hline Metanol & 0 & 5.6 & 88.5 \\
\hline Metanol & 1 & 345.11 & 279 \\
\hline Petróleo crudo & 0 & 12.5 & 83 \\
\hline Petroleo crudo & 1 & 201.2 & 175.8 \\
\hline Etanol & 0 & 18 & 84.3 \\
\hline Etanol & 1 & 104.86 & 161 \\
\hline
\end{tabular}

\section{CONCLUSIONES}

Se aislaron 15 bacterias y una levadura resistentes a petróleo, con el potencial para degradarlo. Además en presencia de petróleo crudo como fuente de carbono presentan una gran actividad de alcohol oxidasa, la cual puede utilizar metanol, etanol y petróleo crudo como sustratos, por lo cual pueden utilizarse para eliminar el hidrocarburo presente en aguas y suelos contaminados. 


\section{REFERENCIAS}

Alvarado-Caudillo, Y. y otros 6 autores. Presence and physiologic regulation of alcohol oxidase activity in an indigenous fungus isolated from petroleum-contaminated sites. Applied Biochemistry and Biotechnology. 98-100, 243-255 (2002).

Argumedo-De Lira, R. y otros 3 autores. El género fúngico Trichoderma y su relación con contaminantes orgánicos e inorgánicos. Revista Internacional de Contaminación ambiental. 25 (4), 257-269 (2009).

Atlas, M.R y otros 3 autores, Response of microbial population to environmental disturbance. Microbiology Ecology. 22, 249-256 (1991).

Auffret, M. y otros 4 autores. Degradation of a mixture of hydrocarbons, gasoline, and diesel oil additives by Rhodococcus aetherivorans and Rhodococcus wratislaviensis. Applied and Environmental Microbiology. 5(24), 7774-7782 (2009).

Chen, S. y C. Lioao. Health risk assessment on human exposed to environmental polycyclic aromatic hydrocarbons pollution sources. Science Total Environmental. 366, 112-123 (2006).

Durón-Castellanos A. y otros 5 autores. Detection of NAD+-dependent alcohol dehydrogenase activities in YR-1 strain of Mucor circinelloides, a potential bioremediator of petroleum contaminated soils. Applied Biochemistry and Biotechnology. 121, 121-124 (2005).

Emtiazi, G., H. y otros 3 autores. Utilization of petroleum hydrocarbons by Pesudomonas sp. and transformed Escherichia coli. African Journal of Biotechnology. 4(2) 172-176 (2005).

Husaini, A., y otros 3 autores. Biodegradation of aliphatic hydrocarbon by indigenous fungi isolated from used motor oil contaminated sites. World Journal Microbiology Biotechnology. 24, 2789-2797 (2008).

Ilori, M.O., A. A. Sunday y C. O. Adedoyin. Isolation and characterization of hydrocarbondegrading and biosurfactant-producing yeast strains obtained from a polluted lagoon water. World Journal Microbiology Biotechnology. 24, 2539-2545 (2008).

Koneman, E. W. y otros 5 autores. Diagnóstico Microbiológico. Texto y Atlas color, $5^{\mathrm{a}}$. Ed. Editorial Médica Panamericana. Buenos Aires. 203-250. (2002).

Leahy, J.G. y R.R. Colwell. Microbial degradation of hydrocarbons in the environment. Microbiology Review. 54, 305-315 (1990).

Lee, K.L., H.R. Buckely y C.C. Campbell. An amino acid liquid synthetic medium for the development of mycelial and yeast forms of Candida albicans. Journal of Medicine and Veterinary Micology. 13, 145-153 (1975).

López Martínez, R. y otros tres autores, Hongos contaminantes comunes en el Laboratorio. En Micología Médica. Procedimientos para el diagnóstico de Laboratorio, 2a . Ed. Trillas. pp 137-148. México (2004).

Lowry, O.H. y otros 3 autores. Protein measurement with the Folin phenol reagent. Journal of Biological Chemistry 193, 265-275. (1951).

Martín Moreno, C., A. González Becerra y M.J. Blanco Santos. Tratamientos biológicos de suelos contaminados: contaminación por hidrocarburos: Aplicaciones de hongos en tratamientos de biorrecuperación. Revista Iberoamericana de Micología. 21, 103-120 (2004). 
Mittal, A. y P. Singh. Isolation of hydrocarbon degrading bacteria from soils contaminated with crude oil spills. Indian Journal of Experimental Biology. 47, 760-765 (2009).

Rivera Cruz, M.C., y otros 4 autores. Adaptación y selección de microorganismos autóctonos en medios de cultivo enriquecidos con petróleo crudo. Terra. 423-434 (2002).

Saadoum, I., y otros 3 autores. Growth of Streptomyces spp. from hydrocarbon-polluted soil and their analysis for the presence of alkane hydroxylase gene (alkB9 by PCR. World Journal Microbiology Biotechnology. 24, 2191-2198 (2008).

Silva-Jiménez, H. y otros 5 autores. Intracellular distribution of fatty alcohol oxidase activity in Mucor circinelloides YR-1 isolated from petroleum cantamianted soils. Antonie van Leewenhoek. 96, 527-535 (2009).

Sugiera, K., M. y otros 3 autores. Physicochemical properties and biodegradability of crude oil. Environmental Science Technolology 31, 45-51 (1997).

Tang, X., Y. Zhu y Meng, Q. Enhanced crude oil biodegradability of Pseudomonas aeruginosa ZJU after preservation in crude oil-containing medium. World Journal Microbiology Biotechnology. 23(7), 7-14 (2007). 